\title{
Internet and the support of psychology education
}

\author{
ANNIE TRAPP, NICK HAMMOND, and DAN BRAY \\ University of York, York, England
}

\begin{abstract}
The Computers in Teaching Initiative (CTI) Centre for Psychology has been promoting the use of computers in psychology education for the last 5 years. The advent of the Internet and ubiquitous access to the World-Wide Web has the potential to revolutionize the way the teaching and learning of psychology can be supported. This paper describes how we are using the Web to support psychology education through information dissemination, through the provision of teaching resources, and through facilities for communication.
\end{abstract}

The changing demands on higher education systems necessitate a reconsideration of how education is delivered and how students learn. This is true in the United Kingdom as much as in the United States. About 5 years ago, a growing realization that educational technology might provide one route to enable attitudes, practices, and organizational structures to change led the U.K. higher education funding bodies to invest resources in building up an infrastructure to support and encourage the exploitation of educational technology. Although this infrastructure, the Computers in Teaching Initiative (CTI), was originally intended as a short-term "pump-priming" exercise, CTI proved to be so successful that it has become an established part of higher education in the U.K., with funding promised until 1999 and likely to continue thereafter. The Initiative provides support through 23 discipline-based centers charged with promoting the use of learning technology within their own disciplines. Until recently, information has been provided to the various disciplines through conventional means; however, the burgeoning use of the Internet has opened a new medium for CTI. This paper focuses on the use of the World-Wide Web by one CTI Centre, the CTI Centre for Psychology; we call upon our experiences to discuss how the Internet can serve as an innovative vehicle for improving and extending the use of learning technologies in psychology education through the provision of three key elements: information, resources, and communication.

To set the context, we first briefly describe the work of the CTI Centre for Psychology.

Correspondence should be addressed to A. Trapp, CTI Centre for Psychology, University of York, Heslington, York YO1 5DD, England (e-mail: ctipsych@york.ac.uk).

\section{The CTI Centre for Psychology}

The psychology community in the U.K. is small by U.S. standards: The Centre supports 160 psychology departments and about 2,000 psychology lecturers. The staff complement of the Centre is a full-time manager, a part-time assistant, and a part-time secretary.

Since its inception about 5 years ago (Hammond \& Trapp, 1991), the Centre has been able to gain a unique overview of the use of learning technology in U.K. psychology departments (see Trapp \& Hammond, 1994, for a report of a recent survey). The role of the Centre, to inform lecturers about technology-based teaching resources and their effective use, is realized through a variety of information dissemination and other activities, including paper publications, visits, demonstrations, workshops, and a biennial conference. We produce a newsletter three times a year, Psychology Software News, focusing mainly on reviews of software, and an annual directory of software relevant to psychology teaching, The CTI Directory of Psychology Software, containing descriptions of about 600 packages. In addition, we maintain a large archive of software at the CTI Centre, which is open for inspection by visitors to the Centre.

The growing interest in the use of learning technologies combined with the amount of relevant information poses two problems for the Centre. First, the variety of information we provide is becoming increasingly difficult to coordinate and update. Second, the number and the nature of queries we receive overstretch our resources. From the start we have tried to facilitate a network of expertise by putting enquirers in touch with others who have tackled similar problems; now, however, it is more feasible to set up a wider range of ways for the psychology community to share information and resources in a more evenly distributed fashion. 
The use of the Internet has enabled the Centre to seek new solutions to these problems both by enabling more effective means of providing integrated and timely information and by affording additional means for effective communication and sharing among institutions.

\section{Support Through Information Dissemination}

In 1994 the Centre launched its own WWW site, and a primary use has been to make information about extant resources more widely available. By accessing on-line information, a lecturer is able, for example, to identify an item of software by searching the Web version of the CTI directory, immediately calling up an associated review, and perhaps downloading a demonstration version of the software to try out. Information about price, publishers, and so forth, is also available. Links are also provided to related services, such as ComPsych at Plattsburgh or iec ProGAMMA in Groningen, The Netherlands. For us, information is easily added or updated; for users, it is more accessible, more up-to-date, and more integrated. In addition, the Web provides a useful forum for dialogue. We have also started using the WWW to obtain feedback from users regarding their views and requirements.

The great majority of departments of psychology in the U.K. provide access to the Internet, and many but not all lecturers have direct access to the Web. Certainly evaluation of our Web server has revealed wide usage both nationally and internationally, particularly of the software directory. A number of lecturers, however, whether by choice or by necessity, do not make use of Web browsers, and so we still (at least at the time of writing) produce our newsletter and directory in paper form. Indeed, it might be argued that, despite the large audience for electronically based information, high-quality paper-based materials still have an impact and influence that electronic information has been unable to achieve.

\section{Support Through Provision of Resources}

An extension to providing information about resources for use in psychology teaching is facilitating direct access to resources. Until recently we have achieved this through demonstrations and tutorials at workshops and by showing available software to visitors to the Centre. Generally in U.K. psychology teaching there is a growing interest in resource-based learning, fueled, perhaps, by the increases in student numbers especially at the introductory level. The Internet offers an additional means for providing and accessing resources for a range of teaching and learning activities, and indeed such resources are starting to become available over the Internet, although as yet these are somewhat sparse for mainstream psychology teaching and serve more as exemplars of future intent than of current use.

Although providing access to resources or materials via the Web, whether these will be used interactively or downloaded for local use, can be effective, it may often be better to provide additional technological, educational, or organizational support so that materials can be seen within an educational context, and perhaps adapted for particular requirements. In putting together a course, a lecturer typically draws on a variety of sources to incorporate within the set of instructional and learning activities that contribute to the students' experiences; lecturers have a good deal of autonomy, and at times idiosyncracy, in course design. In our own use of the Web for providing resources, we have attempted to offer resources together with such support information, and where possible we have sought additional feedback or evaluations from users of the materials.

One example of such a resource is a library of experiments for use with experiment generator software that we have recently set up. Virtually all psychology departments in universities make use of computers to run laboratory experiments for undergraduate practical classes, and many are now developing computer-based practicals using experiment generator software, such as MEL, ERTS, SuperLab, or PsyScope. Quite apart from the wastefulness of individuals in different institutions all reinventing the same wheel, it makes practical sense for materials developed for these packages to be more widely available. We have therefore set up a service whereby library members can submit experiments to the library and access experiments provided by others. Experiments submitted to the library are validated and must have appropriate technical and educational support documentation. They can also include additional information such as data sets collected using the software. Lecturers who make use of an item in teaching are required to complete a short evaluation form so that information about use of the item grows and is available to subsequent users. In a similar fashion, it would be possible to associate an electronic discussion or news group for lecturers and students with an entry in the library, although we have not yet explored this possibility. Our focus has been on teaching applications of experiment generators; the library could well be extended to cover research applications focusing on the accurate replication of experimental procedures and on the reuse and adaptation of experimental methods and techniques.

\section{Support Through Peer and \\ Expert Communication}

The foregoing example leads to the third area of educational support for which the Web has potential: the enhancement of learning through communication associated with the use of resources. The use of communication technologies in learning is a growing research area, and there is particular interest in the role that so-called tertiary courseware (materials created during the course of learning that can themselves be reused in later learning) may play in technology-based learning (Mayes, 1995). Whether in terms of supporting dialogue reuse or more generally in terms of supporting elaboration and discussion about a topic of interest, the Internet provides innovative opportunities for the generation and use of tertiary courseware.

We have recently started a project in this area to support the learning of psychology and can give two brief examples. The first we have termed party pieces. Most teach- 
ers have at least one exemplary lecture or presentation with high-quality support materials and a well-practiced and finely honed script. Lecturers are often more than willing to spend a little time working with us to make their party pieces more widely available in the form of short multimedia presentations. Such materials are not themselves tertiary courseware, but can form the basis for the creation of questions, answers, and discussions, which are. We plan to make party piece presentations available as Web resources and to include both additional support information and an associated discussion list led by one of the authors or another expert who can answer queries or provide further information.

The second example elaborates on the first. It is based on the Answer Garden concept (Ackerman, 1992). Ackerman's Answer Garden allows questions, together with answers from experts, to be structured within a database that subsequent students can browse to see, for example, whether their queries have already been answered. A student can submit a question for which there is not yet a satisfactory answer in the database, and this is automatically routed to an appropriate expert, and then inserted, together with the answer, into the database (provided the question is deemed by the expert to be of sufficient interest). In this way the database grows with real use. An elaboration of the Answer Garden is to add a simple conferencing facility to provide a discussion forum for students and lecturers that is based on the evolving hierarchical structure of questions and answers. The Web, together with any one of a number of proprietary systems that now support simple conferencing and question-and-answer structures, would be an ideal vehicle for this application. We plan to explore the educational practicalities of this application soon.

\section{Conclusion}

We have discussed our experiences and proposals for the use of the Internet to extend support for psychology education through the dissemination of information; through the generation of, and access to, resource materials; and through enhancing communication among the participants in learning. These three areas of application are not independent learning activities but are complementary and interdependent, and indeed they tend to be brought together under the umbrella of facilities provided by the Web. Current technology provides opportunities for enhancing the learning of psychology and for responding to the changing needs of education - opportunities that were not even envisaged 5 years ago. We need to exploit those opportunities now, even if we cannot foresee the possibilities lying beyond the horizon.

\section{REFERENCES}

ACKERMAN, M. (1992). Answer Garden and the organization of expertise (Center for Co-ordination Science Tech. Rep. No. 127). Cambridge: Massachusetts Institute of Technology, Center for Co-ordination Science.

Hammond, N. V., \& TrapP, A. L. (1991). Computers in psychology teaching in the UK. Behavior Research Methods, Instruments, \& Computers, 23, 118-120.

MAYES, J. T. (1995). Learning technology and Groundhog Day. In W. Strang, V. B. Simpson, \& D. Slater (Eds.), Hypermedia at work. Practice and theory in higher education (pp. 21-37). Canterbury: University of Kent.

TRAPP, A. L., \& Hammond, N. V. (1994). The use of educational technology in UK psychology departments. Social Science Computing Review, 12, 519-539.

(Manuscript received November 13, 1995; accepted for publication November 20, 1995.) 